\title{
In-Situ Growth of Passivation Oxide Layer on Fe-Nanoparticle under Electron-Beam Irradiation in a TEM
}

\author{
C. M. Wang ${ }^{*}$, D. R. Baer ${ }^{*}$, J. E. Amonette ${ }^{*}$, M. H. Engelhard ${ }^{*}$, J.J. Anthony ${ }^{* *}$, and Y. Qiang ${ }^{* *}$ \\ *Pacific Northwest National Laboratory, P.O. Box 999, Mail Stop K8-93, Richland, WA \\ 99352 \\ **Physics Department, University of Idaho, Moscow ID 83844-0903
}

A pristine surface of $\mathrm{Fe}^{0}$ exposed to air or an oxygen-including atmosphere will be oxidized instantly by a process normally called initial oxidation. This initial oxidation will lead to an instant formation of an oxide layer on the metal surface. Nanometer-sized iron particles exposed to oxygen-including environments show no exclusion from this rule. This leads to the Fe nanoparticles being covered by a thin layer of oxide to form core-shell structured Fe nanoparticles. The typical thickness of this oxide layer is $\sim 2-3$ $\mathrm{nm}$ [1]. In the Cabrera and Mott model of initial oxidation (or later variations) the further layer growth is inhibited when electrons cannot tunnel through the oxide [2]. In this paper, we report our observation of continued thickening of the native oxide layer on a $\mathrm{Fe}$ nanoparticle as a result of electron-beam bombardment of the particle in a transmission electron microscope (TEM). Estimates of the temperature increases due to the electronbeam bombardment of the specimen fall within the range of $\sim 8 \mathrm{~K}$. Thus, the effect of temperature increase on the continued oxidation is thought to be negligible. Instead, it is proposed that, in accordance with the Cabrera-Mott initial oxidation theory, the thickening of this oxide layer is attributed to the build up of charge on the oxide layer due to a high secondary electron emission by the surface oxide layer. The build up of charge in this layer alters the ability of electrons and ions to migrate through the film and enables the oxidation process to continue. Since iron metal is readily oxidized at typical microscope vacuum conditions, the effect of the charge buildup is to allow oxidation by altering charge transport through the otherwise oxide layer.

An important prediction of the Cabrera-Mott theory is that if there is a way to re-establish the electrical field between the metal and the oxide layer, then the oxide film will be thicker than that normally observable following room temperature passivation of the metal surface. In a recent book, Fromm [3] suggested an experiment in which an electron-beam bombardment of the oxide surface would lead to a condition such that a high oxide rate will be maintained and a thicker oxide layer will be formed. This is exactly what we have observed and reported in this paper.

The Fe nanoparticles used in this study were prepared using gas phase vaporization methods and the particles were subsequently exposed to an oxidizing environment to make an iron core-oxide shell structure. The core-shell structured Fe-iron oxide nanoparticles were analyzed using high resolution transmission electron microscopy (HRTEM) imaging, electron-energy-loss spectroscopy (EELS), and selected-area electron diffraction (SAED) combined with computer simulation. Fig. 1 shows a particle with a lateral dimension of $\sim 25 \mathrm{~nm}$. Initially, this particle has an oxide layer of $\sim 2.38$ $\mathrm{nm}$ in thickness. After 2 hours of electron-beam bombardment, the thickness of the oxide layer increased to $\sim 6 \mathrm{~nm}$ (an increase of $3.62 \mathrm{~nm}$ ), and correspondingly, the dimension of the Fe core decreased by $0.88 \mathrm{~nm}$. The oxide layer is dominated by the $\mathrm{Fe}_{3} \mathrm{O}_{4} / \gamma-\mathrm{Fe}_{2} \mathrm{O}_{3}$ and maintains an invariable orientation relationship of $\mathrm{Fe}[001] / /$ oxide[110] and $\mathrm{Fe}(-$ 
200)//oxide(004). Differentiation of $\mathrm{Fe}_{3} \mathrm{O}_{4}$ from $\gamma-\mathrm{Fe}_{2} \mathrm{O}_{3}$ is not possible solely based on electron diffraction determination of lattice constant. Using bcc Fe as an internal standard with a lattice constant of $\mathrm{Fe}=2.8664 \AA$, the lattice constant of the oxide layer determined from electron diffraction equals to $8.0665 \AA$. This is smaller than the lattice constant of both $\mathrm{Fe}_{3} \mathrm{O}_{4}(0.83958 \AA)$ and $\gamma-\mathrm{Fe}_{2} \mathrm{O}_{3}(0.83457 \AA)$.

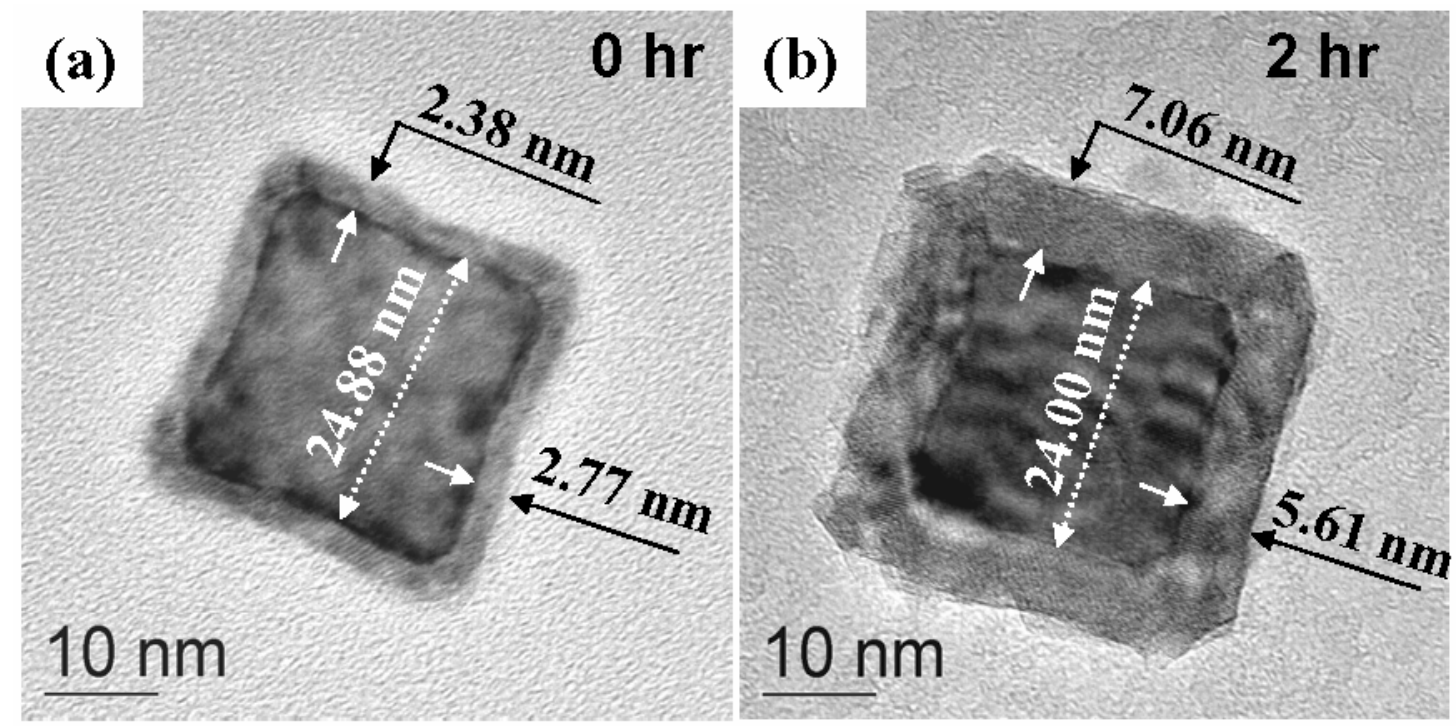

Fig. 1. Thickening of the oxide layer under electron-beam bombardment. (a) $0 \mathrm{hr}$ and (b) after $2 \mathrm{hr}$.

The increases in thickness for the in-situ grown oxide layer are out of proportion with the decreases in the dimension of the Fe core. Based on mass conservation and supposing the phase of the oxide formed is $\mathrm{Fe}_{3} \mathrm{O}_{4}$ having cubic morphology, the decrease of the $\mathrm{Fe}$ core dimension, $\Delta \mathrm{Fe}$, and the corresponding increase of the oxide layer, $\Delta \mathrm{Fe}_{3} \mathrm{O}_{4}$, should satisfy $\Delta \mathrm{Fe}_{3} \mathrm{O}_{4}=1.28 \Delta \mathrm{Fe}$ following the $3 \mathrm{Fe}+2 \mathrm{O}_{2} \leftrightarrow \mathrm{Fe}_{3} \mathrm{O}_{4}$ reaction. Our observations show that the increase of the shell thickness is almost twice that predicted by this simple calculation. This discrepancy is at least partially related to the formation of voids at the interface between the metal and the oxide [1].

Acknowledgements: This work was supported by the U.S. Department of Energy (DOE) Office of Energy Research, Offices of Basic Energy Sciences and Biological and Environmental Research. Parts of the work were conducted in the William R. Wiley Environmental Molecular Sciences Laboratory (EMSL) a DOE User Facility operated by Battelle for the DOE Office of Biological and Environmental Research. Pacific Northwest National Laboratory is operated for the DOE under Contract DE-AC0676RLO 1830.

\section{References}

[1] C. M. Wang, D. R. Baer, L. E. Thomas, J. E. Amonette, J. Antony, Y. Qiang, and G.Duscher, 094308 (2005). J. Appl. Phys. 98, 094308 (2005).

[2] N. Cabrera and N. F. Mott, Rep. Prog. Phys. 12, 163 (1947-1948).

[3] E. Fromm, Kinetics of Metal-Gas Interactions at Low Temperature: Hydriding, Oxidation, Poisoning (Springer, Berlin, Heidelberg, New York, 1998). 